\title{
Alcohol inhibits and disinhibits sexual behavior in the male rat
}

\author{
JAMES G. PFAUS and JOHN P. J. PINEL \\ University of British Columbia, Vancouver, British Columbia, Canada
}

\begin{abstract}
Anecdotal evidence from human case studies suggests that alcohol exerts opposite effects on sexual behavior: facilitation at low doses and disruption at higher doses. We tested this dosedependent dual-effect hypothesis in Experiment 1 by assessing the effects of a wide range of doses of alcohol $(0.25$ to $2.0 \mathrm{~g} / \mathrm{kg}$, i.p.) on the copulatory behavior of sexually active male rats. Rather than the predicted dual effect, all doses of alcohol disrupted copulation, with the highest blocking it completely. Because the facilitatory effect of alcohol on human sexual behavior is believed to reflect a release from inhibition, we hypothesized that the facilitatory effect of low doses of alcohol might be found only when sexual responding was inhibited. In Experiment 2, we tested this hypothesis by assessing the effects of two doses of alcohol $(0.5$ and $1 \mathrm{~g} / \mathrm{kg})$, both of which had proven disruptive in Experiment 1, on the sexual behavior of male rats that had learned to inhibit their sexual behavior during tests with sexually nonreceptive females. Following injection with the lower dose, most of the previously nonresponsive males mounted the nonreceptive females and ejaculated without ever gaining vaginal intromission. These results provide the first experimental evidence that alcohol can inhibit or disinhibit the copulatory behavior of male rats depending upon dose and upon the absence or presence, respectively, of sexual inhibition.
\end{abstract}

The effects of alcohol on sexual behavior are of special interest for two diametrically different reasons. On one hand, alcohol consumption has been repeatedly implicated in the etiology of various sexual disorders; and on the other, it has been reported to enhance sexual arousal and behavior. The general purpose of the present experiments was to contribute to the resolution of this apparent paradox.

Although alcohol is widely believed to increase sexual arousal by a process of disinhibition, that is, by inhibiting cortical centers allegedly responsible for sexual inhibition (Carver, 1948; Ford \& Beach, 1951; Hollister, 1975; Kaplan, 1974; Lemere \& Smith, 1973; MacDougald, 1967; Masters, Johnson, \& Kolodny, 1986), empirical reports of the facilitatory effect of alcohol on subjective and physiological measures of human sexual arousal have been inconsistent. Some experiments on males have demonstrated a slight facilitation of certain physiological measures of sexual arousal (e.g., percent of maximum penile tumescence) at low blood alcohol levels but a marked inhibition of the same measures at higher blood alcohol levels (Farkas \& Rosen, 1976; Rubin \& Henson, 1976; Wilson \& Niaura, 1984). In other experiments, however, alcohol has reduced both subjective and physiological measures of male sexual arousal at all active doses (Briddell \& Wilson, 1976; Farkas \& Rosen, 1976; Rubin \& Henson, 1976). In females, alcohol intoxication has been found

The authors wish to thank Bruce Christensen for his help in conducting Experiments 1 and 2 . This research was supported by a grant from the British Columbia Health Care Research Foundation awarded to John P. J. Pinel. Requests for reprints should be sent to J. P. J. Pinel, Department of Psychology, 2136 West Mall, University of British Columbia, Vancouver, British Columbia V6T 1Y7, Canada. to decrease physiological measures of sexual arousal while increasing subjective measures (Wilson \& Lawson, 1976, 1978). Complicating the situation even further is the fact that consuming a nonalcoholic drink that is believed to contain alcohol has been found to increase the subjective sexual arousal of males (Marlatt, Demming, \& Reid, 1973; Wilson, 1977; Wilson \& Lawson, 1976) but not that of females (Wilson \& Lawson, 1978).

Inconsistency also pervades the clinical case-study literature on alcohol and human sexual performance. Numerous reports have linked alcohol use to such sexual dysfunctions as inhibited sexual desire, erectile failure, delayed ejaculation, and inhibited orgasm (Hollister, 1975; Kaplan, 1974; Kinsey, 1966; Lemere \& Smith, 1973; Masters \& Johnson, 1966; McKendry et al., 1983; Pinhas, 1987; Wilson, 1977); however, others have suggested that alcohol consumption can improve sexual performance in some individuals with preexisting sexual dysfunctions, such as premature ejaculation, inhibited sexual desire, or inhibited orgasm (Pinhas, 1987; Smith, Wesson, \& ApterMarsh, 1984).

Only two published experiments exist in which the effects of alcohol on human sexual behavior have been examined directly, one in males (Malatesta, Pollack, Wilbanks, \& Adams, 1979) and one in females (Malatesta, Pollack, Crotty, \& Peacock, 1982). In both of these experiments, the effects of a range of doses of alcohol were examined on subjects attempting to masturbate to orgasm while viewing a sexually explicit film. In males, alcohol dose-dependently delayed ejaculation, reduced the intensity of sexual orgasm, and decreased both physiological and subjective measures of sexual arousal. Most of these effects were also observed in females: alcohol dose- 
dependently decreased physiological measures of sexual arousal, delayed orgasm, and decreased the subjective intensity of orgasm. However, the females reported that alcohol increased their subjective levels of sexual arousal. Although the results of these experiments support clinical observations that alcohol can disrupt sexual activity, their relevance to the reported effects of alcohol on copulatory behavior is unclear.

Another approach to clarifying the nature of alcohol's effect on sexual performance has been to assess its effect on the sexual behavior of laboratory animals copulating under controlled conditions. Moderate to high doses of alcohol have been shown to delay or block erection, ejaculation, and mounting behavior in both male dogs and rats (Dewsbury, 1967; Gantt, 1940, 1952, 1957; Hart, 1968, 1969; Teitelbaum \& Gantt, 1958). Unfortunately, the effect of low doses of alcohol on the copulatory behavior of laboratory animals has not been assessed, nor has there been any attempt to document alcohol's putative disinhibitory effect on sexual behavior. The objective of the present study was to take advantage of the experimental control offered by an animal model to test hypotheses about the effects of alcohol on sexual behavior derived from the human literature.

\section{GENERAL METHODS}

\section{Subjects and Surgery}

Male Long-Evans and female Sprague-Dawley rats were obtained from Charles River Canada, Inc., St. Constant, Quebec. They were housed by sex in groups of 6 in standard wire-mesh cages in a colony room maintained at approximately $21^{\circ} \mathrm{C}$ on a reversed $12: 12-\mathrm{h}$ light:dark cycle. Food and water were continuously available in the home cages. Twenty-four female rats, which served as stimulus females in both experiments, were bilaterally ovariectomized, via lumbar incisions, under ether anesthesia, approximately 1 month prior to Experiment 1 . The stimulus females were divided into two cohorts of 12 , and each cohort served on alternate test sessions. To render the females sexually receptive, we injected each with $10 \mu \mathrm{g}$ of estradiol benzoate $48 \mathrm{~h}$ before each test and $500 \mu \mathrm{g}$ of progesterone $4 \mathrm{~h}$ before each test.

\section{Drug Treatments}

Ethyl alcohol (95\%) was diluted with physiological saline to obtain doses of $0.25,0.5,1.0$, and $2.0 \mathrm{~g} / \mathrm{kg}$, each in a $25 \%$ aqueous $\mathrm{v} / \mathrm{v}$ solution. The control solution of physiological saline was administered in a volume equal to that of the highest dose of ethanol. Both the ethanol solutions and the saline vehicle were injected intraperitoneally (i.p.) $45 \mathrm{~min}$ before each test. The dose and time-course parameters were chosen on the basis of evidence showing that blood alcohol levels in rodents reach a peak approximately 60 to $90 \mathrm{~min}$ after i.p. injections of a 25\% v/v alcohol solution (Goldstein, 1983). Estradiol benzoate and progesterone (Steraloids) were dissolved in $0.1 \mathrm{ml}$ of peanut oil and injected subcutaneously (s.c.).

\section{Testing Procedure}

Both Experiments 1 and 2 began with 10 baseline tests of copulatory behavior. All baseline tests were $45 \mathrm{~min}$ long and were conducted in a dimly lit room in $29 \times 45 \mathrm{~cm}$ Plexiglas testing chambers lined with San-i-Cel bedding. The baseline tests occurred once every 4 days during the middle third of the dark phase of the circadian light:dark cycle. Each male was habituated to the testing chamber for 5 min prior to the introduction of a sexually receptive female, which marked the beginning of the test session.
For all tests, the occurrence of each mount, intromission, and ejaculation was entered by an experienced observer on a computerized event recorder, which subsequently calculated the following seven primary measures of masculine copulatory behavior: (1) mount and (2) intromission latencies (times from the introduction of the female to the first mount and the first intromission); (3) ejaculation latency (time from the first intromission to the first ejaculation); (4) the postejaculatory interval (time from the first ejaculation to the next intromission); (5) the number of mounts and (6) the number of intromissions prior to the first ejaculation; and (7) the total number of ejaculations. Calculated from these seven primary measures were two secondary measures: the interintromission interval (the ejaculation latency/number of intromissions) and the intromission rate (the number of mounts with intromission/total number of mounts with and without intromission).

\section{EXPERIMENT 1}

In contrast to the numerous anecdotal reports that low doses of alcohol can facilitate human sexual behavior, experiments in laboratory animals had revealed only disruption. We hypothesized that alcohol might facilitate the sexual behavior of animals at doses lower than those used in previous experiments (i.e., at doses lower than $0.5 \mathrm{~g} / \mathrm{kg}$ ). We tested this hypothesis in Experiment 1 by assessing the effects of a wide range of doses of alcohol on the copulatory behavior of sexually active male rats.

\section{Method}

Subjects. The subjects were 60 sexually active 400-600-g LongEvans male rats.

Procedure. Following the 10 baseline copulatory tests, the rats were assigned randomly to one of five alcohol-dose groups $(0,0.25$, $0.5,1.0$, or $2.0 \mathrm{~g} / \mathrm{kg})$. The rats in each group $(n=12)$ received an i.p. injection of alcohol $45 \mathrm{~min}$ before the 45 -min copulation test, which occurred 4 days after the last baseline test and was identical to it.

The significance of the differences in the proportion of rats in each group that displayed mounts, intromissions, or ejaculations was assessed using chi-square analyses. The effects of alcohol on the seven primary and two secondary measures of copulatory behavior were subjected to nonparametric Kruskal-Wallis analyses of variance for independent measures $(p<.05)$. For each measure of copulatory behavior found to be significantly affected by the alcohol treatment, multiple pairwise comparisons were made between the effect of each dose of alcohol and the effect of saline using nonparametric Mann-Whitney tests. Using the Bonferroni method (Marascuilo \& Levin, 1983), all multiple comparisons were corrected for elevated experiment-wise error.

\section{Results}

Alcohol produced a dose-dependent disruption of male copulatory behavior. The two lowest doses, 0.25 and $0.5 \mathrm{~g} / \mathrm{kg}$, produced a moderate degree of disruption, which was reflected by changes in only a few of the measures. The disruption produced by the $1-\mathrm{g} / \mathrm{kg}$ dose was greater and more extensive than that produced by the two lower doses, and the highest dose $(2 \mathrm{~g} / \mathrm{kg})$ resulted in a complete lack of sexual activity. There was no evidence whatsoever of the hypothesized facilitatory effect at any dose.

As illustrated in Figure 1, alcohol significantly decreased the proportion of rats that mounted $\left[\chi^{2}(4)=\right.$ $13.41, p<.01]$, intromitted $\left[\chi^{2}(4)=13.41, p<.01\right]$, 

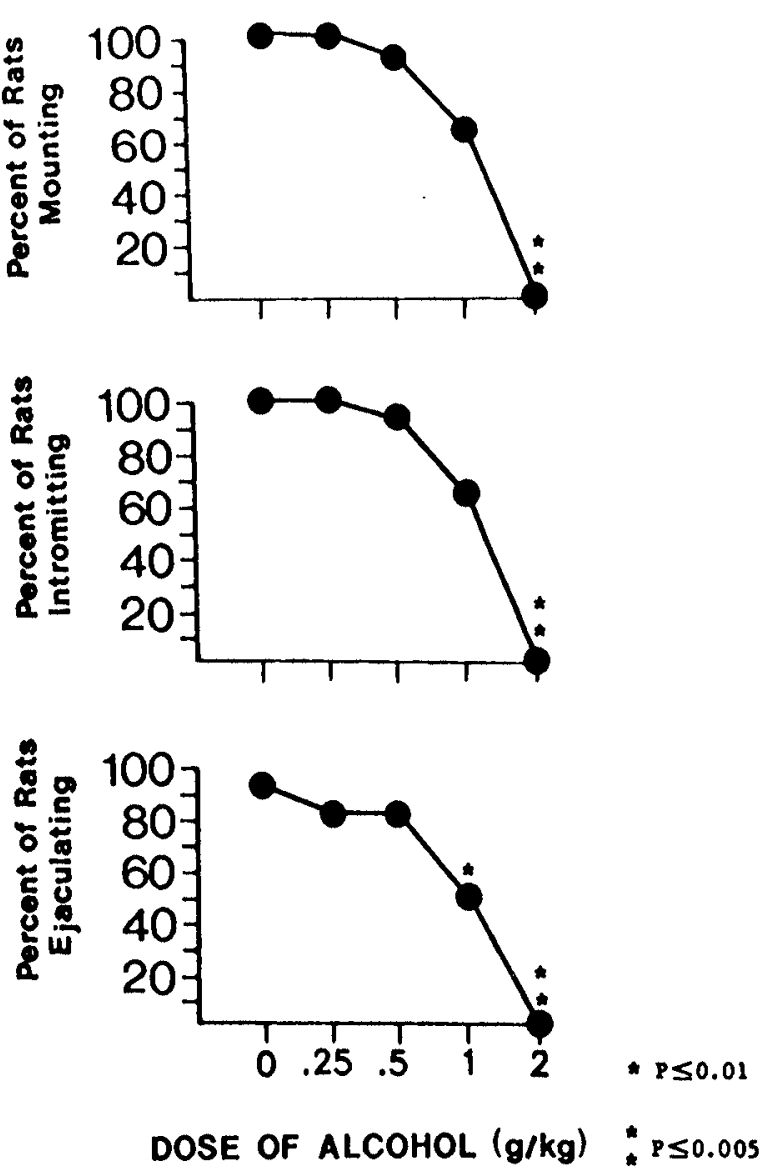

Figure 1. Percentage of rats displaying mounts, intromissions, and ejaculations as a function of alcohol dose in Experiment 1.

and ejaculated $\left[\chi^{2}(4)=15.74, p<.01\right]$. Post hoc pairwise comparisons of the proportion of subjects that mounted, intromitted, and ejaculated in each alcohol group and the proportions in the saline control group revealed that the effects of the two lower doses were not statistically significant. In contrast, the $1-\mathrm{g} / \mathrm{kg}$ dose significantly reduced the proportion of rats that ejaculated $\left[\chi^{2}(1)=\right.$ $5.56, p<.01]$, and although it substantially reduced the proportion of rats that mounted and intromitted, these latter effects did not reach statistical significance with the alpha adjusted by the Bonferroni method (both chisquare $p s<.05$ but $>.01$ ). The rats that received the highest dose $(2 \mathrm{~g} / \mathrm{kg})$ of alcohol were conspicuously ataxic, and none attempted copulation during the 45-min test (all three chi-square $p s<.0001$ ).

The effects of different doses of alcohol on the primary and secondary measures of copulatory behavior were compared statistically using only the data of those rats in each group that displayed the copulatory behavior under analysis. For example, statistical analyses of the differences in latencies to mount, intromit, and ejaculate were based on the data of only those rats that mounted, intromitted, and ejaculated, respectively; no arbitrary scores were assigned. The effects of alcohol on these measures are shown in Table 1.

In rats that engaged in copulatory behavior, alcohol significantly increased the latencies to first mount $(H=8.65$, $p<.05)$, first intromission $(H=10.27, p<.025)$, and first ejaculation $H=11.82, p<.01$ ). In addition, the number of intromissions preceding the first ejaculation was significantly increased by alcohol treatment $(H=$ $16.73, p<.005$ ), and alcohol significantly decreased the number of ejaculations achieved during the 45 -min test $(H=25.83, p<.001)$. Although alcohol markedly increased the number of mounts without intromission, this effect did not reach statistical significance $(H=6.23$, $p<.10$ ). Finally, in rats that copulated, alcohol had no significant effect on the postejaculatory interval, the interintromission interval, or the intromission rate.

Post hoc comparisons revealed that the $1-\mathrm{g} / \mathrm{kg}$ dose of alcohol significantly increased the mount latency $(U=$ $18.5, p<.01)$ and the intromission latency $(U=16$, $p<.01$ ) of those rats that mounted and intromitted, respectively. Although the $0.5-\mathrm{g} / \mathrm{kg}$ dose also produced a substantial increase in the latencies to first mount and intromission, these effects did not reach the statistical sig-

Table 1

Effects of Alcohol on Male Sexual Behavior

\begin{tabular}{|c|c|c|c|c|c|c|c|c|c|c|}
\hline \multirow[b]{3}{*}{ Behavioral Parameters } & \multicolumn{10}{|c|}{ Dose of Alcohol } \\
\hline & \multicolumn{2}{|c|}{$\begin{array}{c}0 \mathrm{~g} / \mathrm{kg} \\
\text { (Control) }\end{array}$} & \multicolumn{2}{|c|}{$.25 \mathrm{~g} / \mathrm{kg}$} & \multicolumn{2}{|c|}{$.50 \mathrm{~g} / \mathrm{kg}$} & \multicolumn{2}{|c|}{$1.0 \mathrm{~g} / \mathrm{kg}$} & \multicolumn{2}{|c|}{$2.0 \mathrm{~g} / \mathrm{kg}$} \\
\hline & $M$ & $S E$ & $M$ & $S E$ & $M$ & $S E$ & $M$ & $S E$ & $M$ & $S E$ \\
\hline Mount latency & 47.8 & 15 & 57.9 & 20 & 154.1 & $47^{*}$ & 270.0 & $81 \dagger$ & - & \\
\hline Intromission latency & 75.5 & 16 & 103.6 & 35 & 186.2 & $42^{*}$ & 340.4 & $78 \dagger$ & - & \\
\hline Ejaculation latency & 540.5 & 143 & 838.4 & 155 & 1039.4 & $130 \dagger$ & 1341.5 & $144 \dagger$ & - & \\
\hline Postejaculatory interval & 386.4 & 18 & 357.9 & 13 & 418.1 & 32 & 378.2 & 8 & - & \\
\hline Number of mounts & 13.3 & 3 & 14.1 & 4 & 17.6 & 2 & 24.1 & 4 & - & \\
\hline Number of intromissions & 4.8 & 1 & 12.3 & $2 \dagger$ & 8.8 & $1 \dagger$ & 9.3 & $1 \dagger$ & - & \\
\hline Number of ejaculations & 1.8 & .2 & 1.4 & 3 & 1.1 & $.2 *$ & 0.8 & $.2 \dagger$ & - & \\
\hline Interintromission interval & 152.3 & 69 & 104.3 & 47 & 328.4 & 161 & 146.7 & 14 & - & \\
\hline Intromission rate & 0.34 & .06 & 0.52 & .05 & 0.32 & .04 & 0.30 & .02 & - & \\
\hline
\end{tabular}

Note-Table 1 presents the effects of each dose of alcohol on the seven primary and two secondary measures of sexual behavior assessed in Experiment 1. The means and standard errors are from rats that displayed the appropriate copulatory behavior in each group. All latencies and intervals are in seconds. The number of mounts and intromissions are calculated prior to the first ejaculation. ${ }^{*} p<.05, \dagger p<.01$, and $\ddagger p<.005$ from control values. 
nificance required by the Bonferroni adjustment (both $p s<.05$ but $>.01$ ). In contrast, the ejaculation latencies of those rats that ejaculated were increased significantly by both the $0.5-\mathrm{g} / \mathrm{kg}$ dose $(U=23.5, p<.01)$ and the $1-\mathrm{g} / \mathrm{kg}$ dose $(U=12, p<.01)$. The number of intromissions preceding ejaculation was increased significantly by the $0.25-\mathrm{g} / \mathrm{kg}$ dose $(U=12.5, p<.01)$, the $0.5-\mathrm{g} / \mathrm{kg}$ dose $(U=24, p<.01)$, and the $1-\mathrm{g} / \mathrm{kg}$ dose $(U=4, p<.01)$. Finally, although each dose of alcohol substantially decreased the number of ejaculations in rats that copulated, the decrease was statistically significant only at the $1-\mathrm{g} / \mathrm{kg}$ dose $(U=22.5, p<.01)$.

\section{Discussion}

The results of Experiment 1 confirmed previous reports that moderate to high doses of alcohol disrupt or block copulatory behavior in sexually active male rats (Dewsbury, 1967; Hart, 1968, 1969), but they did not confirm our hypothesis that low doses of alcohol facilitate copulatory behavior. Moderate to high doses of alcohol (i.e., 1 and $2 \mathrm{~g} / \mathrm{kg}$ ) substantially decreased the proportion of rats that mounted, intromitted, and ejaculated. Moreover, in rats that engaged in copulatory behavior, low to moderate doses (i.e., 0.5 and $1 \mathrm{~g} / \mathrm{kg}$ ) increased the mean latencies to first mount, first intromission, and first ejaculation, and reduced the total number of ejaculations that they achieved during the $45-\mathrm{min}$ test. Although the $1-\mathrm{g} / \mathrm{kg}$ dose significantly increased the mean number of intromissions, which might, at first glance, seem to be evidence of facilitation, an increase in the number of intromissions required to trigger an ejaculation is widely considered to be evidence of disruption or desensitization (see Sachs \& Barfield, 1976).

Several hypotheses can be advanced to account for the present results. The two most obvious are that alcohol produced either a general disruption of motor activity or a general aversive state incompatible with sexual behavior. The first hypothesis could account for the ataxia observed in rats injected with the highest dose. Although this hypothesis could also account for some of the disruptive effects observed at lower doses (e.g., the decreased percentage of rats that initiated or completed an ejaculatory series), it cannot account for other effects (e.g., the increased number of intromissions that preceded ejaculation). Furthermore, none of the rats that copulated to ejaculation following alcohol treatment appeared sluggish in its general activity or in its attempts to pursue the stimulus females. Although we cannot rule out the second hypothesis, that alcohol might have disrupted sexual behavior by inducing an incompatible aversive state, we note that i.p. injections of alcohol at doses lower than $1.75 \mathrm{~g} / \mathrm{kg}$ are not sufficient to induce conditioned taste aversions in rats (Jeffreys, Pournaghash, \& Riley, 1989).

There are three possible explanations for the disruption of sexual activity that occurred at the lower doses: (1) alcohol may have reduced sexual motivation, (2) it may have interfered with the perception of stimuli essential for eliciting sexual activity, or (3) it may have produced a selective disruption of the motor responses involved in copulation. Although it was not the purpose of this experiment to distinguish among these three interpretations, our results provide some support for the first two over the last. Diminished sexual motivation can be inferred from several of the observed effects, including the decreased proportion of rats that initiated mounts and intromissions, the increased mount and intromission latencies of rats that eventually copulated, and the increased ejaculation latencies of rats that eventually ejaculated. The same effects, together with the increased number of intromissions, can also be taken as evidence that alcohol desensitized the rats to such external cues as estrous odors, female proceptive behaviors, or penile stimulation. In contrast, alcohol did not reduce the intromission ratio or increase the interintromission interval. Thus, there is little support in the present results for the idea that alcohol disrupts copulatory behavior by reducing the ability of rats to perform sexual responses. We tentatively suggest that low to moderate doses of alcohol disrupt male copulatory behavior by reducing sexual motivation, by reducing sensitivity to sexual stimulation, or by some combination of the two.

\section{EXPERIMENT 2}

In Experiment 1, all doses of alcohol disrupted the copulatory behavior of sexually active male rats. Despite the numerous anecdotal reports that low doses of alcohol can facilitate human sexual behavior, no evidence of facilitation was observed at any dose. There are two possible explanations for this inconsistency. First, alcohol may affect the sexual behavior of rodents differently than it does the sexual behavior of humans. In this regard, Wilson (1977) has argued against the relevance of animal models to study the effects of alcohol on human sexual behavior. A critical feature of Wilson's argument is his assumption that lower animals lack the "cognitive mediation" commonly associated with the control of sexual behavior in humans. If alcohol facilitates human sexual behavior by an action on cognitive processes specific to human sexual behavior, then attempts to generalize the effects of alcohol on rodent sexual behavior to human sexual behavioror, as in the present case, from human to rodent-have little merit.

The second explanation for the absence of facilitatory effects in Experiment 1 stems from the traditional belief that alcohol facilitates sexual behavior by releasing it from inhibitory control. Accordingly, it is possible that low doses of alcohol failed to facilitate sexual behavior in Experiment 1 , not because the effects of alcohol on sexual behavior are fundamentally different in rodents and humans, but because the sexual behavior of the male rats was not under inhibitory control. The purpose of Experiment 2 was to test this disinhibition hypothesis.

To determine whether alcohol can facilitate the sexual behavior of male rats when it is under inhibitory control, we first induced sexually active male rats to suppress their copulatory behavior by giving them repeated tests with 
sexually nonreceptive females (Pfaus, Jacobs, \& Wong, 1986). We then assessed the effects of a low $(0.5 \mathrm{~g} / \mathrm{kg})$ and a moderate $(1.0 \mathrm{~g} / \mathrm{kg})$ dose of alcohol, both of which had disrupted copulatory behavior in Experiment 1, on the sexual behavior of the inhibited males.

\section{Method}

Subjects. The subjects were 30 sexually active $400-600-\mathrm{g}$ LongEvans male rats.

Procedure. Experiment 2 began with 10 baseline tests identical to those of Experiment 1. Following these baseline tests, each rat was trained to inhibit its sexual activity during tests with nonreceptive females. During this training phase, the male rats received seven 45-min tests with sexually nonreceptive females (i.e., inhibitory tests), one every 4 days. Two days prior to each of these seven inhibitory tests, the males received a similar 45 -min test with sexually receptive females (i.e., a noninhibitory test). To habituate them to the injection procedure, all of the male rats received a saline injection $(2 \mathrm{ml} / \mathrm{kg}$ ) $45 \mathrm{~min}$ before each of the 14 tests of the training phase.

Following the training phase was a drug-testing phase comprising four additional tests: two noninhibitory tests and two inhibitory tests administered on the same alternating 2-day schedule established during the training phase. Prior to the first test (noninhibitory) of the drug-testing phase, the rats were randomly assigned to one of three drug conditions $(n=10)$ and received either saline, $0.5 \mathrm{~g} / \mathrm{kg}$, or $1 \mathrm{~g} / \mathrm{kg}$ of alcohol $45 \mathrm{~min}$ before the test. All rats received saline prior to the two intervening tests. On the fourth and final test (inhibitory) of the drug-testing phase, the rats once again were randomly assigned to one of the three drug conditions $(n=10)$ and injected accordingly $45 \mathrm{~min}$ before the test. This method made it possible to compare the effects of different doses of alcohol in the same group of rats under conditions of sexual excitation and inhibition.

The data were analyzed as in Experiment 1 . However, because the male rats could not gain vaginal intromission during the test with nonreceptive females (i.e., during the inhibitory test), statistical comparisons involving the inhibitory test focused exclusively on mount and ejaculation measures.

\section{Results}

Development of sexual inhibition during the training phase. Consistent with the results of Pfaus et al. (1986), the male rats gradually learned to suppress their copulatory behavior during a series of regularly scheduled tests with nonreceptive females while maintaining their baseline rates of copulatory behavior during intervening tests with receptive females. Figure 2 illustrates the proportion of rats that displayed mounts and ejaculations during the seven inhibitory tests and seven noninhibitory tests that composed the training phase of Experiment 2. The monotonic decline in the proportion of rats that attempted mounts during the inhibitory tests is readily apparent: On the first inhibitory test, $80 \%$ of the rats mounted the nonreceptive females, but on the fifth, sixth, and seventh inhibitory tests, none of the rats attempted copulation. In marked contrast, all 30 rats mounted and ejaculated during each of the seven noninhibitory tests.

Effects of alcohol during inhibitory and noninhibitory tests. The major result of Experiment 2 is clearly evident in Figure 3. During the inhibitory test, the $0.5-\mathrm{g} / \mathrm{kg}$ dose of alcohol significantly increased the proportion of rats that mounted $\left[\chi^{2}(2)=7.91, p<.005\right]$ and ejaculated $\left[\chi^{2}(2)=5.95, p<.01\right]$, despite the fact that the
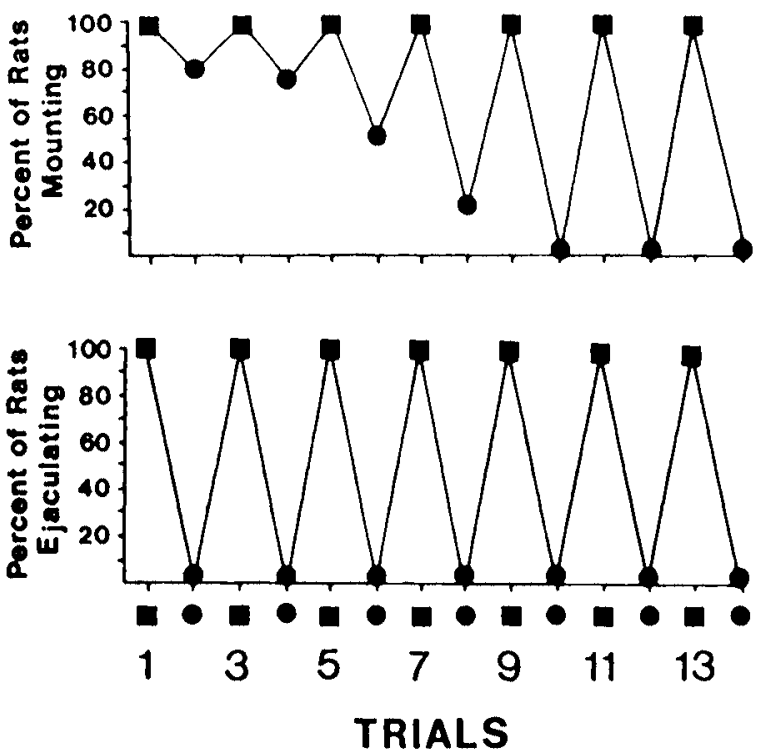

Figure 2. Percentage of rats displaying mounts or ejaculations during the training phase of Experiment 2. Squares represent tests with sexually receptive females; circles represent tests with nonreceptive females.

nonreceptive females were uncooperative. Although 2 of the rats that received the $1-\mathrm{g} / \mathrm{kg}$ dose of alcohol attempted to mount the nonreceptive females, this increase above the saline baseline was not statistically significant. Neither of these 2 rats ejaculated.

The effects of alcohol during the noninhibitory test confirmed the results of the comparable drug tests administered in Experiment 1 . The $1-\mathrm{g} / \mathrm{kg}$ dose of alcohol sig-

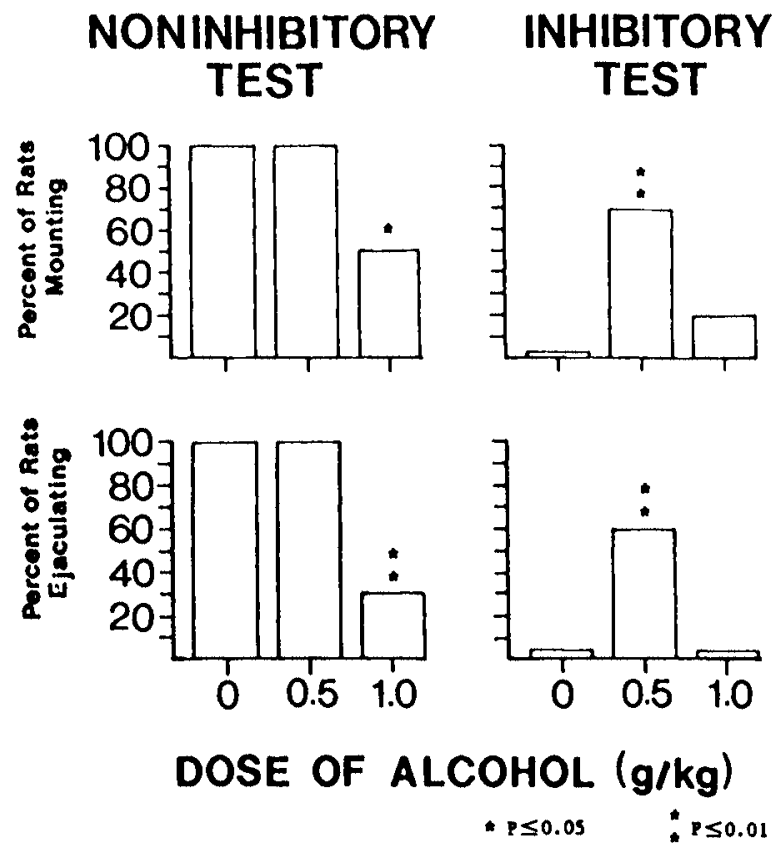

Figure 3. Percentage of rats displaying mounts or ejaculations as a function of alcohol dose during the noninhibitory and inhibitory tests in Experiment 2. 
nificantly reduced the proportion of rats that mounted $\left[\chi^{2}(2)=4.27, p<.025\right]$ and ejaculated $\left[\chi^{2}(2)=7.91\right.$, $p<.005]$, whereas the $0.5-\mathrm{g} / \mathrm{kg}$ dose did not. Furthermore, as in Experiment 1 , the $1-\mathrm{g} / \mathrm{kg}$ dose significantly increased the mount, intromission, and ejaculation latencies, and increased the number of mounts and intromissions that preceded ejaculation; and the $0.5-\mathrm{g} / \mathrm{kg}$ dose significantly increased the ejaculation latency and the number of mounts and intromissions that preceded ejaculation (all $p$ s $<.01$ ).

Performance during the two intervening baseline tests of the drug-testing phase indicated that there had been no shift in baseline. None of the rats mounted or ejaculated during the intervening inhibitory test, whereas all rats mounted and ejaculated during the intervening noninhibitory test.

\section{Discussion}

In Experiment 2, a low dose of alcohol $(0.5 \mathrm{~g} / \mathrm{kg})$, which disrupted but did not block the copulatory behavior of male rats during a test with sexually receptive females, released copulatory behavior from the inhibition induced by regular tests with sexually nonreceptive females. A higher dose of alcohol $(1 \mathrm{~g} / \mathrm{kg})$, sufficient to disrupt or block the copulatory behavior of male rats during a noninhibitory test, was not effective in releasing copulatory behavior from inhibition during an inhibitory test.

These results are consistent with anecdotal reports that low doses of alcohol can facilitate human copulatory behavior through a process of disinhibition (Athanasiou, Shaver, \& Tavris, 1970; Hollister, 1975; Lemere \& Smith, 1973; Masters et al., 1986). They are also consistent with results showing that low doses of alcohol that decrease physiological measures of sexual arousal in human males under conditions of sexual excitation can impair the ability of human males to voluntarily inhibit their sexual arousal (Rubin \& Henson, 1976; Wilson \& Niaura, 1984). These similarities challenge Wilson's (1977) contention that the effects of alcohol on the sexual behavior of laboratory animals provide few insights into the effects of alcohol on human sexual activity. It would be of interest in future studies to determine whether doses of alcohol lower than $0.5 \mathrm{~g} / \mathrm{kg}$ can facilitate the copulatory behavior of males given training with sexually nonreceptive females and to assess the effect of alcohol on other conditions of low sexual activity (e.g., in sexually sluggish males or in males trained to inhibit their sexual advances by different means).

\section{GENERAL DISCUSSION}

The present experiments were designed to investigate alcohol's putative inhibitory and facilitatory effects on the copulatory behavior of sexually active male rats under conditions of sexual excitation and inhibition. The results confirmed that a low dose of alcohol can facilitate or disrupt copulatory behavior in male rats depending upon the presence or absence, respectively, of sexual inhibition.

The theory proposed by Steele and Southwick (1985) to account for the disinhibitory effects of alcohol on human social behavior adapts well to the present results. This theory suggests that alcohol disinhibits behavior because intoxicated individuals lack the perceptual cognitive functioning necessary to inhibit inappropriate responses in the face of strong eliciting cues. The theory predicts that the disinhibitory effect of alcohol can occur only in those situations in which there is strong inhibitory conflict, that is, in situations in which inhibitory control is in conflict with strong behavior-eliciting cues. Accordingly, in Steele and Southwick's terms, the low dose of alcohol facilitated copulation in Experiment 2 because there were both strong eliciting cues and inhibitory control. However, we can only speculate about the identity of the eliciting stimuli in this condition. Because the female rats were not sexually receptive, none of the potential stimulus qualities specifically associated with proceptivity or receptivity (e.g., pheremonal cues, soliciting behaviors, or lordosis) were present. When the males attempted to mount, the nonreceptive females generally displayed such defensive behaviors as biting, boxing, kicking, running in circles, and lying on their backs. It is conceivable that simply encountering a female, regardless of its state of estrus, in a testing chamber associated with copulation, might have been a sufficient eliciting cue for copulation. It is also possible, however, that the intoxicated rats misinterpreted the females' defensive behavior for proceptive behavior. Darting, kicking, and running in circles are characteristics of both types of behavior (Beach, 1976; Madlafousek \& Hlinak, 1978).

The disinhibitory effects of alcohol are not restricted to sexual behavior. In mice, equivalent doses of alcohol potentiate suppressed aggression against an intruder in an unfamiliar environment but reduce the display of unsuppressed aggression against an intruder in the home cage (Miczek \& O'Donnell, 1980). In rats, equivalent doses of alcohol reverse the suppressive effects of corticotropinreleasing factor (CRF) on punished responding for food but act synergistically with CRF to inhibit unpunished responding for food (Thatcher-Britton \& Koob, 1986). And in humans, equivalent doses of alcohol decrease the amount of ice cream eaten by nondieters but increase the amount eaten by dieters (Polivy \& Herman, 1976).

In summary, the present study provides the first experimental evidence that alcohol can both disrupt and disinhibit the copulatory behavior of male rats. The fact that these results were predicted from a consideration of anecdotal reports of alcohol's effects on human sexual activity lends support to the disinhibitory theory of alcohol's facilitatory effects on human sexual behavior and underscores the value of the comparative approach in the study of drug-induced sexual disinhibition. 


\section{REFERENCES}

Athanasiou, R., Shaver, P., Tavris, C. (1970). Sex. Psychololgy Today, 4, 39-52.

BEACH, F. A. (1976). Sexual attractivity, proceptivity, and receptivity in female mammals. Hormones \& Behavior, 7, 105-138.

BridDELL, D. W., W WLSON, G. T. (1976). Effects of alcohol and expectancy set on male sexual arousal. Journal of Abnormal Behavior, 85, 225-234.

CARver, A. E. (1948). The interrelationship of sex and alcohol. International Journal of Sexology, 2, 78-81.

DEWSBURY, D. A. (1967). Effects of alcohol ingestion on copulatory behavior of male rats. Psychopharmacologia (Berlin), 11, 276-281.

FARKAS, G. M., \&OSEN, R. C. (1976). Effect of alcohol on elicited male sexual response. Journal of Studies on Alcohol, 37, 265-272.

Ford, C. S., \& BEACH, F. A. (1951). Patterns of sexual behavior. New York: Harper \& Row.

GANTT, W. H. (1940). Effect of alcohol on sexual reflexes in dogs. American Journal of Physiology, 129, 360.

GANTT, W. H. (1952). Effect of alcohol on the sexual reflexes of normal and neurotic male dogs. Psychosomatic Medicine, 14, 174-181.

GANTT, W. H. (1957). Acute effect of alcohol on automatic (sexual, secretory, cardiac) and somatic responses. In H. E. Himwich (Ed.), Alcoholism: Basic aspects and treatment (pp. 73-89). Washington, DC: American Association for the Advancement of Science.

Goldstein, D. B. (1983). Pharmacology of alcohol. New York: Oxford University Press.

HART, B. L. (1968). Effects of alcohol on sexual reflexes and mating behavior in the male dog. Quarterly Joumal of Studies on Alcohol, 29, 839-844.

HART, B. L. (1969). Effects of alcohol on sexual reflexes and mating behavior in the male rat. Psychopharmacologia (Berlin), 14, 377-382.

Hollister, L. E. (1975). The mystique of social drugs and sex. In M. Sandler \& G. L. Gessa (Eds.), Sexual behavior: Pharmacology and biochemistry (pp. 85-92). New York: Raven Press.

Jeffreys, R., Pournaghash, S., \& Riley, A. L. (1989). Conditioned taste aversions to alcohol in rats. Manuscript submitted for publication.

Kaplan, H. S. (1974). The new sex therapy. New York: Brunner.

KINSEY, B. A. (1966). The female alcoholic. Springfield, IL: Thomas.

LEMERE, F., \& SMITH, J. W. (1973). Alcohol-induced sexual impotence. American Joumal of Psychiatry, 130, 212-213.

MacDougaLD, D. (1967). Aphrodesiacs and anaphrodesiacs. In A. Ellis \& A. Abarbanel (Eds.), Encyclopaedia of sexual behavior (pp. 145153). New York: Hawthorn Books.

Madlafousek, J., \& HuinaK, Z. (1978). Sexual behavior of the female rat: Inventory, patterning, and measurement. Behavior, 63, 129-173.

Malatesta, V. J. , Pollack, R. H., Crotty, T. D., Peacock, L. J. (1982). Acute alcohol intoxication and female orgasmic response. Journal of Sex Research, 18, 1-17.

Malatesta, V. J., Pollack, R. H., Wilbanks, W. A., \& Adams, H. E. (1979). Alcohol effects on the orgasmic-ejaculatory response in human males. Journal of Sex Research, 15, 101-107.
Marascuilo, L., \&evin, J. (1983). Multivariate statistics in the social sciences: A researcher's guide. Monterey, CA: Brooks/Cole.

Marlatt, G. A., Demming, B., \& Reid, J. B. (1973). Loss of control drinking in alcoholics: An experimental analogue. Journal of $A b$ normal Psychology, 81, 233-241.

Masters, W. H., Johnson, V. E. (1966). Human sexual response. Boston: Little-Brown.

Masters, W. H., Johnson, V. E., \& Kolodny, R. C. (1986). Sex and human loving. Boston: Little-Brown.

McKendry, J. B. R., Colluns, W. E., Silverman, M., Krul, L. E., Collins, J. P., \& Irvine, A. H. (1983). Erectile impotence: A clinical challenge. Canadian Medical Association Journal, 128, 653-663.

MiczeK, K. A., \& O'Donnell, J. M. (1980). Alcohol and chlordiazepoxide increase suppressed aggression in mice. Psychopharmacology, 69, 39-44.

Pfaus, J. G., Jacobs, W. J., \& Wong, R. (1986). Conditional olfactory cues facilitate the acquisition of copulatory behavior and affect mate choice in male rats. Canadian Psychology, 27, 470.

Pinhas, V. (1987). Sexual dysfunctions in women alcoholics. Medical Aspects of Human Sexuality, 21, 97-101.

Polivy, J., \&erman, C. P. (1976). Effects of alcohol on eating behavior: Influence of mood and perceived intoxication. Jourmal of $A b$ normal Psychology, 85, 601-606.

Rubin, H. B., \& Henson, D. E. (1976). Effects of alcohol on male sexual responding. Psychopharmacology, 47, 123-134.

SACHS, B. D., \& BARFIELD, R. J. (1976). Functional analysis of masculine copulatory behavior in the rat. Advances in the Study of Behavior, 7, 91-154.

Smith, D. E., Wesson, D. R., A Apter-Marsh, M. (1984). Cocaineand alcohol-induced sexual dysfunctions in patients with addictive disease. Journal of Psychoactive Drugs, 16, 359-361.

STEELE, C. M., SoutHWICK, L. (1985). Alcohol and social behavior: I. The psychology of drunken excess. Joumal of Personality \& Social Psychology, 48, 18-34.

Teitelanum, H. A., Gantt, W. H. (1958). The effect of alcohol on sexual reflexes and sperm count in the dog. Quarterly Journal of Studies on Alcohol, 19, 394-398.

Thatcher-Britton, K., KoOB, G. F. (1986). Alcohol reverses the proconflict effect of corticotropin-releasing factor. Regulatory Peptides, 16, 315-320.

WiLson, G. T. (1977). Alcohol and human sexual behavior. Behavior Research \& Therapy, 15, 239-252.

WILSON, G. T., LAWSON, D. M. (1976). Expectancies, alcohol, and sexual arousal in male social drinkers. Journal of Abnormal Psychology, 85, 587-594.

WiLson, G. T., LAwson, D. M. (1978). Expectancies, alcohol, and sexual arousal in women. Journal of Abnomal Psychology, 87, 358-367.

Wilson, G. T., Niaura, R. (1984). Alcohol and the disinhibition of sexual responsiveness. Journal of Studies on Alcohol, 45, 219-224.

(Manuscript received June 6, 1988;

revision accepted for publication December 15,1988 .) 